\title{
Variations in the nature of metal adsorption on ultrathin $\mathrm{Al}_{2} \mathrm{O}_{3}$ films
}

\author{
Alexander Bogicevic and Dwight R. Jennison \\ Surface and Interface Sciences Department, Sandia National Laboratories, Albuquerque, NM 87185-1413, USA
}

(October 16, 1998)

First-principles density-functional calculations are used to study metal adsorption ( $\mathrm{Li}, \mathrm{K}, \mathrm{Y}, \mathrm{Nb}, \mathrm{Ru}$, $\mathrm{Pd}, \mathrm{Pt}, \mathrm{Cu}, \mathrm{Ag}, \mathrm{Au}$, and $\mathrm{Al}$ at 1/3-4 monolayer coverages) atop $5 \AA \mathrm{Al}_{2} \mathrm{O}_{3}$ films on $\mathrm{Al}(111)$. The $\mathrm{Pd}, \mathrm{Pt}, \mathrm{Cu}, \mathrm{Ag}, \mathrm{Au}$, and $\mathrm{Al}$ at $1 / 3-4$ monolayer coverages) atop $5 \mathrm{~A} \mathrm{Al}_{2} \mathrm{O}_{3}$ films on $\mathrm{Al}(111)$. The
oxide-metal bond is ionic at low coverages but, with interesting exceptions, caused by polaritet
at high coverages where the overlayer is metallic. Binding trends are explained in terms of simple concepts. Increasing overlayer thickness can cause the adsorbate-oxide interface structure to chang: $0 \mathrm{EC} / 1998$ and while some metals wet, most do not.

PACS number(s): $71.15 . \mathrm{Mb}, 68.55 .-\mathrm{a}$

Few fields in materials science are so technologically important, yet poorly understood, as metal-ceramic interfaces. Microchip packaging, catalysis support, corrosion and electrical protection, and biophysical implants are a few areas that would benefit from an atomicscale understanding of oxide-metal bonding. Experimental $[1,2]$ and theoretical approaches [3] have been limited by the inherent complexity of oxides. Many different adhesion mechanisms have been proposed, including van der Waals, covalent, ionic, and polarization [3-5], but there is a profound lack of systematic insight. This unsatisfactory situation suggests that any fundamental and methodical understanding of oxide-metal interactions must include a detailed knowledge of the electronic structure at the interface. Density-functional theory (DFT) $[6,7]$ provides an accurate basis for attacking this complex task from first principles, avoiding problems that have rendered several previous oxide calculations disputable (as discussed in Refs. [3,4]). Progress in algorithms and computer power now make this total-energy method feasible for this class of materials at a time when there is a surge of meticulous oxide experiments $[8,9]$.

In this Letter, we report adsorption properties of metal adsorbates, spanning the periodic table (PT), on ultrathin $\mathrm{Al}_{2} \mathrm{O}_{3}$ films on $\mathrm{Al}(111)$. This allows us to study binding trends due to variations in ionic radii, metal interactions, valence configurations, and ionization potentials. We make several contacts with experiment, and address issues such as wettability and epitaxial growth modes. The ultrathin film is directly relevant to understanding corrosion of $\mathrm{NiAl}$ and the $\mathrm{Ni}_{3} \mathrm{Al}$ family of "superalloys". It is a good model system for bulk sapphire [10], and has the additional advantage that it can be characterized with scanning tunneling microscopy (STM) and ionizing probes.

The ionicity of an oxide and the magnitude of adsorbate-induced relaxations largely determine how metal atoms bind to its surface. The Madelung potential in the near-surface region gives a further indication of what bond type is to be expected. In oxides with lack of strict layer-by-layer neutrality, it can be strong enough to ionize an isolated metal atom [4]. In $\mathrm{MgO}, \mathrm{NiO}$, and other oxides with strict layer neutrality, this potential is considerably weaker. Relaxations are small compared with alumina (where they are critically important, see below), and soft van der Waals or stronger covalent oxidemetal bonds have been reported [5]. As the metal coverage increases, adatom-adatom interactions strengthen at the expense of adatom-oxide bonds, and the metal overlayer can literally rise out of the surface and the potentially oxidizing environment. If this effect is large, it can in fact change the bond type [4].

Our calculations are based on DFT $[6,7]$ at the localdensity approximation (LDA) [11] level, using a supercell method (the VASP code) [12]. The one-electron wave functions are expanded in a plane-wave basis with a fairly low energy cutoff of $20 \mathrm{Ry}$, allowed by ultrasoft Vanderbilt pseudo-potentials [13]. To the left in the PT, shallow core electrons are included in the valence. The Kohn-Sham equations are solved iteratively, and the atomic structure is optimized until the forces on all unconstrained atoms are less than $0.03 \mathrm{eV} / \AA$. The surface Brillouin zone of the super-cell ( 3 atoms per layer, $>16$ $\AA$ of vacuum) is sampled using 10 irreducible $k$-points.

The structure of the ultrathin $\mathrm{Al}_{2} \mathrm{O}_{3}$ film on $\mathrm{Al}(111)$ was recently determined within LDA by Jennison and coworkers [10]. It consists of fourfold coordinated $\mathrm{Al}$ ions between close-packed oxygen sublattices, see Fig. 1. This new phase (named $\tau-\mathrm{Al}_{2} \mathrm{O}_{3}$ ) is stabilized by the properties of the interface. Before considering metal adsorption, we confirm the stability of this structure [14] within the (self-consistent) generalized gradient approximation [15] (GGA). Our super-cell has four layers of $\mathrm{Al}(111)$ below the oxide film. The two bottom-most Al layers are fixed at bulk positions. All other atoms are allowed to relax. We place one metal species at a time on the oxide, varying the adsorption site and coverage $(\Theta=1 / 3-4 \mathrm{ML})$.

All metal atoms bind strongly to the oxide at $1 / 3 \mathrm{ML}$, see Table I. Our density-of-states (LDOS) and chargedensity analyses show that the oxide-metal bond is ionic at low coverages, regardless of metal adsorbate [16]. The large variety of chemically very different adsorbates considered here signifies that this result, first seen for $\mathrm{Pt}$ on sapphire [4], is general. The degree of ionicity depends on the respective ionization potential and adsorption height. The lost metal charge is distributed over (mainly the 


\section{DISCLAIMER}

This report was prepared as an account of work sponsored by an agency of the United States Government. Neither the United States Government nor any agency thereof, nor any of their employees, make any warranty, express or implied, or assumes any legal liability or responsibility for the accuracy, completeness, or usefulness of any information, apparatus, product, or process disclosed, or represents that its use would not infringe privately owned rights. Reference herein to any specific commercial product, process, or service by trade name, trademark, manufacturer, or otherwise does not necessarily constitute or imply its endorsement, recommendation, or favoring by the United States Government or any agency thereof. The views and

opinions of authors expressed herein do not necessarily state or reflect those of the United States Government or any agency thereof. 


\section{DISCLAIMER}

Portions of this document may be illegible in electronic image products. Images are produced from the best available original document. 

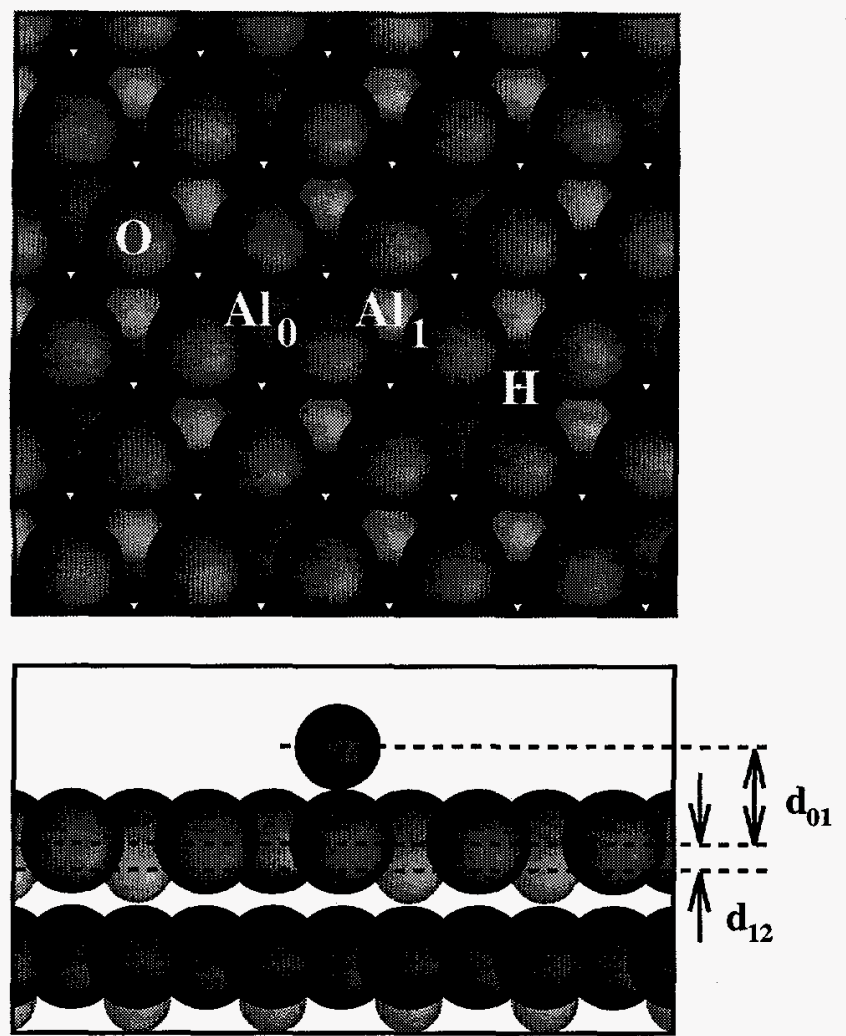

FIG. 1. Top and side views of the $\tau-\mathrm{Al}_{2} \mathrm{O}_{3}$ film with high-symmetry adsorption sites marked. The aluminum ions are drawn smaller.

nearest) neighbor oxygen ions. The metal ions prefer to bind in the three-fold hollow $\left(\mathrm{Al}_{0}\right)$ site, attracted to the $\mathrm{O}^{-2}$ ions [17]. This induces a significant oxide relaxation, which increases the surface polarity (see below).

For a particular site at $1 / 3 \mathrm{ML}$, the adsorption energies increase up and to the left in the PT. The first trend is explained by a decrease in ion size, reducing the adionoxide distance and augmenting the bond. The calculated bond lengths fully confirm this simple picture (Table II). The second trend is ascribed to increasing ionicity to the left within a period, which strengthens the adion-oxide bond despite the fact that the bond length increases, cf., $\mathrm{Ru}-\mathrm{Nb}-\mathrm{Y}$. For equally charged metal ions, the situation is normalized, and the adsorption energies decline with longer bonds, cf., Ru-Pd-Ag. Notice that the binding scales rather nicely with the Pauling electronegativity, save for $\mathrm{K}$. A test calculation with $\mathrm{Li}$ confirms that the exception is due to the large ion size of $\mathrm{K}$ (Tables I-II).

The nature of the oxide-metal bond changes drastically as the coverage is increased. The adsorbates form a metallic overlayer, with weaker bonds to the oxide. Our LDOS and charge-density analyses show that the adhesion at $1 \mathrm{ML}$ is almost purely electrostatic, i.e., with interesting exceptions (see below), the metal overlayer is attracted to the ions at the oxide surface by polarization, irrespective of metal adsorbate. This mechanism, similar to the one proposed by Stoneham [18] and developed by
Finnis et al. [19], was recently found for $\mathrm{Pt}$ adsorption on $\alpha-\mathrm{Al}_{2} \mathrm{O}_{3}$ [4]. At $1 \mathrm{ML}$, the electrostatic bond is supplemented by partially ionic binding for $\mathrm{Nb}(\simeq 0.2$ electron/ $\mathrm{Nb}$ atom), which augments the bond by about $19 \%$ $(0.3 \mathrm{eV})[20]$.

The binding trends at $1 \mathrm{ML}$ are the same as at $1 / 3 \mathrm{ML}$, with decreasing adsorption energies down and to the right in the PT. Whereas the trend within a group still is explained by atom size at $1 \mathrm{ML}$ (as corroborated by the calculated adsorbate-oxide distances), the second tendency is here closely linked to rehybridizations. These are illustrated in Fig. 2, which shows the adsorbate-induced charge density difference plot for $\mathrm{Nb}-\mathrm{Ru}-\mathrm{Pd}$ at $1 \mathrm{ML}$ atop-O adsorption. To strengthen the lateral bonds in forming a metal film, the atomic $d$-shells assume an oval shape. In the case of, e.g., $\mathrm{Ru}$ and $\mathrm{Pd}$, this is manifested by charge transfer from the lateral $d$-orbitals to the largely non-bonding $d_{z}^{2}$-orbital. Upon adsorption on the oxide, the (metal) film-oxide distance is minimized by depleting the metal $d_{z}^{2}$-orbitals and oxygen $p_{z}$-orbitals, thereby strengthening the adsorption bond at the expense of lateral metal binding. The resulting polarization seen in Fig. 2 is lateral, making the adsorbate positive above the $\mathrm{O}$ ions and negative between. Metal atoms with less than a half-full $d$-shell, however, rehybridize in the opposite direction when forming a metal film, i.e., depleting their $d_{z}^{2}$-orbitals. The oxygen polarization therefore goes in the opposite direction upon adsorption of $\mathrm{Nb}$ and other metals to the left of Mo (which already have more of their core exposed). Together with the ionicbond supplement, this explains the strong binding for $\mathrm{Nb}$ at $1 \mathrm{ML}$ recently found in experiment [21]. Thus $\mathrm{Nb}$ binds strongly without strong polarization.

Metals adsorb on top of the O or Al sublattice at $1 \mathrm{ML}$. The site preference can in fact be understood in terms of the stiffness (e.g., shear modulus) of the pure metals.

TABLE I. LDA adsorption energies in eV (per atom, with respect to the isolated slab and metal) for various metals in different sites at varying coverage. Preferred sites are highlighted, and unstable adsorption is indicated by a dash. The metallic radius $\epsilon$, normalized to $\mathrm{Al}(1.43 \AA)$, is given together with the Pauling electronegativity $F$ and the calculated ionic charge $q$ at $\Theta=1 / 3 \mathrm{ML}$ (rounded off to the nearest integer).

\begin{tabular}{l|cccc|ccc|ccc|ccc}
\hline \hline & \multicolumn{9}{|c|}{$\Theta=1 / 3$} & \multicolumn{3}{|c|}{$\Theta=2 / 3$} & \multicolumn{3}{|c|}{$\Theta=1$} & & \\
& $\mathrm{Al}_{0}$ & $\mathrm{Al}_{1}$ & $\mathrm{O}$ & $\mathrm{H}$ & $\mathrm{Al}$ & $\mathrm{O}$ & $\mathrm{H}$ & $\mathrm{Al}$ & $\mathrm{O}$ & $\mathrm{H}$ & $\epsilon$ & $F$ & $q$ \\
\hline $\mathrm{Li}$ & $\mathbf{6 . 0}$ & & & & & & & & & & 1.08 & 1.0 & +1 \\
$\mathrm{~K}$ & $\mathbf{3 . 6}$ & 2.4 & 2.4 & - & - & - & - & - & - & - & 1.64 & $0.8+1$ \\
\hline $\mathrm{Y}$ & $\mathbf{6 . 9}$ & 5.2 & 4.2 & - & $\mathbf{3 . 2}$ & 2.4 & 2.9 & $\mathbf{2 . 6}$ & 1.7 & 1.6 & 1.25 & $1.3+3$ \\
$\mathrm{Nb}$ & $\mathbf{6 . 5}$ & 4.9 & 3.8 & - & 2.1 & $\mathbf{2 . 6}$ & 2.4 & $\mathbf{1 . 3}$ & 1.9 & 1.2 & 1.02 & $1.6+2$ \\
$\mathrm{Ru}$ & $\mathbf{5 . 3}$ & 3.6 & 2.8 & - & 1.5 & 1.9 & 1.3 & 0.9 & $\mathbf{1 . 4}$ & 0.6 & 0.94 & $2.2+1$ \\
$\mathrm{Pd}$ & $\mathbf{3 . 2}$ & 1.8 & 1.9 & - & 1.2 & $\mathbf{1 . 2}$ & $\mathbf{1 . 0}$ & $\mathbf{0 . 7}$ & $\mathbf{0 . 7}$ & 0.5 & 0.96 & $2.2+1$ \\
$\mathrm{Pt}$ & $\mathbf{3 . 3}$ & 1.9 & 2.6 & - & $\mathbf{1 . 3}$ & 1.0 & 0.6 & $\mathbf{0 . 6}$ & 0.4 & 0.3 & 0.97 & $2.2+1$ \\
\hline $\mathrm{Cu}$ & $\mathbf{4 . 6}$ & 2.3 & 2.2 & - & $\mathbf{1 . 5}$ & $\mathbf{1 . 4}$ & - & 0.8 & $\mathbf{0 . 9}$ & 0.6 & 0.90 & $1.9+1$ \\
$\mathrm{Ag}$ & $\mathbf{3 . 1}$ & 1.7 & 1.6 & - & $\mathbf{1 . 0}$ & 0.8 & 0.8 & $\mathbf{0 . 6}$ & 0.5 & 0.4 & 1.01 & $1.9+1$ \\
$\mathrm{Au}$ & $\mathbf{2 . 3}$ & $\mathbf{1 . 1}$ & 1.4 & - & $\mathbf{0 . 7}$ & 0.5 & 0.5 & $\mathbf{0 . 4}$ & 0.3 & 0.3 & 1.02 & $2.4+1$ \\
\hline $\mathrm{Al}$ & $\mathbf{5 . 9}$ & 3.7 & 3.6 & - & $\mathbf{2 . 2}$ & 2.1 & 1.6 & $\mathbf{1 . 6}$ & 1.8 & $\mathbf{1 . 1}$ & 1.00 & $1.5+1$ \\
\hline \hline
\end{tabular}




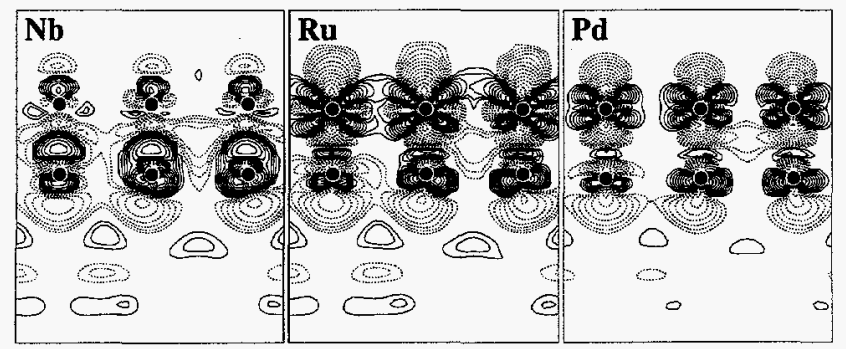

FIG. 2. Adsorbate-induced charge density difference plot for $\mathrm{Nb}, \mathrm{Ru}$, and $\mathrm{Pd}$. Solid lines indicate charge accumulation, dashed lines depletion, with logarithmic increments. The $\langle 100\rangle$ cut goes through the center of the adsorbates and oxygen ions. Note the difference in polarization between metals on different sides of Mo in the PT. The chemisorbed oxygens fall outside of this cut, but polarize in a similar way to the surface oxygens.

Atop-Al adsorption severely buckles the metal film because a third of the metal adatoms lack an $\mathrm{Al}$ ion underneath. This buckling requires more energy for harder metals, which is why these in general prefer atop-oxygen adsorption (without buckling). At $\Theta>1 / 3 \mathrm{ML}$, there is a large strain in the metal overlayer for $\mathrm{Y}$ and $\mathrm{Cu}$, caused by the supercell approach. It is likely that $\mathrm{Y}$ in particular forms an incommensurate overlayer, or experiences some lattice rotation that affects the surface registry [22]. One should therefore be cautious in interpreting some of the high-coverage results for $\mathrm{Y}$ and $\mathrm{Cu}$.

An interesting metal in this regard is potassium. It saturates at $1 / 3 \mathrm{ML}$ coverage (atop the $\mathrm{Al}_{0}$ sites, see Table I), with a K-K distance that is very close to the bulk metal value. This might suggest that $\mathrm{K}$ is metallic at $1 / 3$ ML. However, laser desorption experiments have shown that $\mathrm{K}$ can be removed from sapphire via hot electron attachment [23], which is only possible for non-metallic films. We find that the $\mathrm{K}$ atoms are ionized and do not

TABLE II. Adsorption parameters for preferred binding sites. The notation is described in Fig. 1 , and all values are given in $\AA$. At $1 \mathrm{ML}$, there is a large adsorbate staggering (0.8-2.3 $\AA$ ) for atop-Al binding, so $\mathrm{d}_{01}$ and $\mathrm{d}_{12}$ are average values. Unstable configurations are indicated by a dash.

\begin{tabular}{l|cc|cccc}
\hline \hline & \multicolumn{2}{|c|}{$\Theta=1 / 3$} & \multicolumn{5}{c}{$\Theta=1$} \\
& $\mathrm{~d}_{01}$ & $\mathrm{~d}_{12}$ & $\mathrm{~d}_{01}$ & $\mathrm{~d}_{12}$ & $\mathrm{~d}_{01}$ & $\mathrm{~d}_{12}$ \\
\hline $\mathrm{Li}$ & 0.58 & 0.45 & & & & \\
$\mathrm{~K}$ & 1.74 & 0.50 & - & - & - & - \\
\hline $\mathrm{Y}$ & 1.44 & 0.64 & 3.12 & 0.54 & 2.29 & 0.58 \\
$\mathrm{Nb}$ & 1.34 & 0.58 & 2.48 & 0.45 & 2.11 & 0.56 \\
$\mathrm{Ru}$ & 1.21 & 0.53 & 2.30 & -0.17 & 2.01 & 0.51 \\
$\mathrm{Pd}$ & 1.43 & 0.43 & 2.39 & -0.39 & 2.18 & 0.40 \\
$\mathrm{Pt}$ & 1.44 & 0.43 & 2.41 & -0.40 & 2.26 & 0.36 \\
\hline $\mathrm{Cu}$ & 0.86 & 0.52 & 2.04 & 0.40 & 1.96 & 0.50 \\
$\mathrm{Ag}$ & 1.44 & 0.47 & 2.37 & 0.37 & 2.42 & 0.37 \\
$\mathrm{Au}$ & 1.56 & 0.42 & 2.45 & 0.28 & 2.58 & 0.29 \\
\hline $\mathrm{Al}$ & 1.05 & 0.53 & 2.34 & 0.53 & 1.89 & 0.67 \\
\hline \hline
\end{tabular}

form a metallic overlayer, which explains the experimental observation. Even when we compress the entire lattice a few percent to mimic the bulk metal K-K distance, this result remains intact.

Another intriguing observation concerns the polarity of the oxide surface. The $\mathrm{Al}$ and $\mathrm{O}$ ions in the clean (no adsorbates) relaxed oxide film are nearly coplanar $\left(\mathrm{d}_{12}=0.06 \AA\right)$, thereby neutralizing the surface polarity, as first noted by Jennison et al. [10]. Upon metal adsorption, the relaxation pattern changes dramatically. The oxygen atoms relax outwards by $0.1-0.4 \AA$. This "breathing" is often seen in thin films, and is induced by the presence of the metal. More importantly, the Al ions displace inwards at $\Theta=1 / 3 \mathrm{ML}$, resulting in a large $\mathrm{O}-\mathrm{Al}$ plane distance of $\mathrm{d}_{12}=0.4-0.6 \AA$. This severe relaxation is crucial to the binding. If we freeze all atoms (except the adsorbate) in clean slab positions, the binding typically decreases by $\simeq 2 \mathrm{eV}$. (In contrast, our test calculations for $1 / 8 \mathrm{ML} \mathrm{Pt} / \mathrm{MgO}$ show that relaxation amounts to only $0.2 \mathrm{eV}$ in binding [24]). The $1 / 3 \mathrm{ML}$ relaxation is a result of the small size of $\mathrm{Al}^{+3}$ (cf. $\mathrm{MgO}$ [24]) and the adion- $\mathrm{Al}$ ion repulsion, and further enhances the electrostatic potential at the adsorption site. Consequently, the surface polarity decreases somewhat at $1 \mathrm{ML}$ where the metal overlayer is neutral. The relaxation is driven by polarization, which explains why the effect is smaller for the noble metals, and for atop-O adsorption where the oxygen ions largely screen the electrostatic attraction. The largest relaxation is seen for atop- $\mathrm{Al}$ adsorption of transition metals, where $d_{12}$ even changes sign to the right of Mo (Table II). Our charge-density analyses show that the $\mathrm{Al}$ ions (which do not exhibit any electron rearrangement) simply respond to changes in the electrostatic field caused by the oxygen polarization described above.

An important issue in several technological applications is the degree of wettability and the growth mode of metal overlayers. Kinetic considerations are likely to

TABLE III. Comparison between adsorbate-adsorbate $\left(E_{a}^{a}\right)$ and adsorbate-substrate $\left(E_{s}^{a}\right)$ per-atom free energies $(\mathrm{eV})$. The ratio between these two energies is denoted by $R$. The values are for the most stable site, which is $\mathrm{Al}_{0}$ at $\Theta=1 / 3 \mathrm{ML}$, and $\mathrm{Al}$ or $\mathrm{O}$ at 2/3-1 ML. The Born-Haber cycle energies (see main text) are given in $\mathrm{eV}$.

\begin{tabular}{l|ccc|cccc|cccc|cc}
\hline \hline & \multicolumn{3}{|c|}{$\Theta=1 / 3$} & \multicolumn{5}{|c|}{$\Theta=2 / 3$} & \multicolumn{3}{|c}{$\Theta=1$} & \multicolumn{2}{|c}{$\mathrm{B}-\mathrm{H}$} \\
& $E_{a}^{a}$ & $E_{s}^{a}$ & $R$ & Site & $E_{a}^{a}$ & $E_{s}^{a}$ & $R$ & Site & $E_{a}^{a}$ & $E_{s}^{a}$ & $R$ & $\Delta_{1}$ & $\Delta_{2}$ \\
\hline $\mathrm{Li}$ & 0.8 & 6.0 & 0.1 & & & & & & & & & & \\
$\mathrm{~K}$ & 0.9 & 3.5 & 0.2 & - & - & - & - & - & - & - & - & - & - \\
\hline $\mathrm{Y}$ & 3.2 & 6.9 & 0.5 & $\mathrm{Al}$ & 3.9 & 3.2 & 1.2 & $\mathrm{Al}$ & 4.5 & 2.6 & 1.7 & 7.6 & 6.5 \\
$\mathrm{Nb}$ & 2.0 & 6.5 & 0.3 & $\mathrm{O}$ & 6.6 & 2.6 & 2.6 & $\mathrm{O}$ & 8.6 & 1.9 & 4.5 & -6.9 & -1.1 \\
$\mathrm{Ru}$ & 1.3 & 5.3 & 0.3 & $\mathrm{O}$ & 5.5 & 1.9 & 2.9 & $\mathrm{O}$ & 7.1 & 1.4 & 4.9 & -6.4 & -8.4 \\
$\mathrm{Pd}$ & 1.5 & 3.2 & 0.5 & $\mathrm{Al}, \mathrm{O}$ & 3.6 & 1.2 & 3.0 & $\mathrm{Al}, \mathrm{O}$ & 4.7 & 0.7 & 6.5 & -2.5 & -4.0 \\
$\mathrm{Pt}$ & 0.6 & 3.3 & 0.2 & $\mathrm{Al}$ & 4.1 & 1.3 & 3.2 & $\mathrm{Al}$ & 5.7 & 0.6 & 9.8 & -7.2 & -4.8 \\
\hline $\mathrm{Cu}$ & 0.3 & 4.6 & 0.1 & $\mathrm{Al}$ & 2.1 & 1.5 & 1.4 & $\mathrm{O}$ & 3.0 & 0.9 & 3.4 & 3.5 & -3.9 \\
$\mathrm{Ag}$ & 0.5 & 3.1 & 0.1 & $\mathrm{Al}$ & 2.1 & 1.0 & 2.2 & $\mathrm{Al}$ & 2.9 & 0.6 & 4.7 & 0.1 & -1.5 \\
$\mathrm{Au}$ & 0.4 & 2.3 & 0.2 & $\mathrm{Al}$ & 2.6 & 0.8 & 3.5 & $\mathrm{Al}$ & 3.6 & 0.4 & 10.4 & -4.3 & -1.6 \\
\hline $\mathrm{Al}$ & 1.0 & 5.9 & 0.2 & $\mathrm{Al}$ & 2.6 & 2.2 & 1.2 & $\mathrm{O}$ & 3.0 & 1.8 & 1.7 & 4.9 & 2.6 \\
\hline
\end{tabular}


be significant at low and intermediate temperatures, and are hard to model due to the complex oxide structure and variation of bond type with coverage. A good starting point for growth mode considerations is to look at the thermodynamics through Born-Haber cycles. The relevant measures here are $\Delta_{1}=E_{\mathrm{sys}}^{1 \mathrm{ML}}+2 E_{\mathrm{slab}}-3 E_{\mathrm{sys}}^{1 / 3 \mathrm{ML}}$ and $\Delta_{2}=E_{\mathrm{sys}}^{2 \mathrm{ML}}+E_{\mathrm{slab}}-2 E_{\mathrm{sys}}^{\mathrm{ML}}$ (the subscript "sys" denotes metal+oxide system). $\AA$ positive value of $\Delta_{1}\left(\Delta_{2}\right)$ suggests that the adsorbate will wet the surface (grow in a layer-by-layer fashion), see Table III. A few experimental results have been reported for metal- $\mathrm{Al}_{2} \mathrm{O}_{3}$ systems, which enables us to test the validity of this admittedly simplistic approach. We find that $\mathrm{Cu}$ wets the oxide, but clusters up upon subsequent deposition. This has recently been confirmed in STM and Auger experiments on ultrathin $\mathrm{Al}_{2} \mathrm{O}_{3}$ films on $\mathrm{Ni}_{3} \mathrm{Al}$ by Kelber and coworkers [25]. A similar agreement with experiment is noted for the non-wetting metals Pd and Pt on both sapphire [8] and film [2].

An interesting finding is that the interface structure changes when the metal overlayer grows beyond $1 \mathrm{ML}$. This has a simple explanation: the newly deposited atoms increase the coordination of and thereby stiffen the metal film interface, rendering it more bulk-like. In line with our considerations above, the reluctance to buckle favors atop-O adsorption for all metals except for the soft $\mathrm{Y}$, which prefers atop- $\mathrm{Al}$ adsorption. $\mathrm{Al}$ is in this context neither hard nor soft, and follows the trend by binding equally strong to both sublattices.

Finally, we make a few technical notes. The use of ultra-soft pseudopotentials yields adsorption energies within $0.1 \mathrm{eV}$ of those obtained by using hard potentials or higher energy cutoff ( $30 \mathrm{Ry})$. Gradient corrections (GGA) do not affect the binding mechanism or any of the trends we find. GGA does, however, weaken the metaloxide bond by $0.5-1 \mathrm{eV}$ at $\Theta=1 / 3 \mathrm{ML}$, and by $0.2-0.3$ $\mathrm{eV}$ at $1 \mathrm{ML}$. We are presently looking into the reasons for this. LDA has proven to be very accurate for polarization based interactions [26], but more accurate experimental results are needed to discern which functional in the end is closer to reality. We warn against using GGA corrections to LDA-relaxed oxide systems, so-called post-GGA. We find that it is a poor approximation to self-consistent GGA. This has recently been reported (and explained) for other systems as well [27].

In summary, we present extensive total-energy calculations of metal adsorption on ultrathin $\mathrm{Al}_{2} \mathrm{O}_{3}$ films on $\mathrm{Al}(111)$. We show that the oxide-metal bond is ionic at low coverages, and with a few interesting exceptions, a result of polarization attraction at higher coverages. Bond strengths are interpreted in simple terms such as ionization potentials and ionic radii, providing a transparent picture of the quantitative behavior of oxide-metal bonding. The oxide relaxation is found to be crucial to the metal binding, and the oxide-metal interface structure is found to change with increasing overlayer thickness for several metals. We rationalize these observations, explain a recent laser desorption study of potassium, and predict film wettability and growth modes. Several results have been confirmed experimentally.

We thank Jeff Kelber and Hans-Joachim Freund for stimulating discussions and Peter Feibelman and Bruce Bunker for critical readings of the manuscript. VASP was developed at the Institut für Theoretische Physik of the Technische Universität Wien. Sandia is a multiprogram laboratory operated by Sandia Corporation, a Lockheed Martin Company, for the United States Department of Energy under Contract DE-AC04-94AL85000. This work was partially supported by a Laboratory Directed Research and Development project.

[1] V. E. Heinrich and P. A. Cox, The Surface Science of Metal Oxides, Cambridge University Press (1996).

[2] F. Winkelmann et al., Surf. Sci. 307-309, 1148 (1994).

[3] M. W. Finnis, Condens. Matter 8, 5811 (1996).

[4] C. Verdozzi et al., to appear in Phys. Rev. Lett.

[5] I. Yudanov et al., J. Phys. Chem. B 101, 2786 (1997).

[6] P. Hohenberg and W. Kohn, Phys. Rev. 136, B864 (1964).

[7] W. Kohn and L. J. Sham, Phys. Rev. 140, A1133 (1965).

[8] Charles T. Campbell, Surf. Sci. Rep. 27, 1 (1997).

[9] H.-J. Freund, H. Kuhlenbeck, and V. Staemmler, Rep. Prog. Phys. 59, 283 (1996).

[10] D. R. Jennison et al., submitted.

[11] J. P. Perdew et al., Phys. Rev. B 23, 5048 (1981).

[12] G. Kresse and J. Hafner, Phys. Rev. B 47, 558 (1993); 49, 14251 (1994); 54, 11169 (1996).

[13] D. Vanderbilt, Phys. Rev. B 32, 8412 (1985).

[14] The two metastable films mentioned in Ref. [10] are 0.33 and $0.13 \mathrm{eV}$ higher in energy per unitcell within GGA, close to the LDA values of 0.34 and $0.20 \mathrm{eV}$.

[15] J. P. Perdew et al., Phys. Rev. B 46, 6671 (1992).

[16] Covalency is negligible $(<0.1$ electron by computed atomic-orbital based interatomic LDOS).

[17] Assigning a charge to an ion includes some uncertainty associated with the means of integrating the LDOS.

[18] A. M. Stoneham, Appl. Surf. Sci. 14, 249 (1983).

[19] M. W. Finnis, A. M. Stoneham, and P. W. Tasker, in Metal-Ceramic Interfaces, edited by M. Rühle et al. (Oxford: Pergamon, 1990).

[20] At $4 \mathrm{ML}, \mathrm{Nb}$ binds with $1.6 \mathrm{eV} /$ atom, $0.3 \mathrm{eV}$ weaker than at $1 \mathrm{ML}$.

[21] G. Song et al., Phys. Rev. Lett. 79, 5062 (1998).

[22] Neither case can be investigated at this level of accuracy due to the prohibitively large supercells associated with such calculations. The small strain in the other metal overlayers is to a good approximation unimportant, as it in reality is likely to be relieved by an array of dislocations, leaving large in-registry adhesion regions.

[23] K. Al-Shamery et al, to be published.

[24] D. R. Jennison and A. Bogicevic, to be published.

[25] J. A. Kelber et al., to be published.

[26] D. R. Jennison et al., Phys. Rev. Lett. 77, 4828 (1996).

[27] M. Fuchs et al., Phys. Rev. B 57, 2134 (1998). 